\title{
Comparison of Fixed Range Method and Levey-Jennings Method Used in ELISA Detection Anti-HBc
}

\author{
Ju Deyi, Wang Jiandong, Zhao Xiaomei \\ Clinical lab of Jilin City Center Hospital, Jilin, Jilin, 132013, China
}

\begin{abstract}
In this paper aims at using the two different methods (fixed range method and LeveyJennings method) to study the internal quality control of qualitative ELISA detection anti-HBc and exploring the difference, applicability, effectiveness of the two methods. Method -- the author collects the internal quality control data of hepatitis B core antibody during the 16 months (from October 2015 to February 2017). Fixed range method is used. X2 is upper control limit and X1 is lower control limit. Cut-off value adopts one of the lines. ELISA method detection is two half-andhalf. If $\mathrm{S} / \mathrm{CO} \geq 1$ is positive, 1 is the lower limit of week positive control product. If $\mathrm{S} / \mathrm{CO}<1$ is positive, 1 is upper limit of week positive control product. When the quality control product is stable, $\mathrm{X}+3 \mathrm{SD}$ or $\mathrm{X}-3 \mathrm{SD}$ of $\mathrm{S} / \mathrm{CO}$ value shall be included into Levey-Jennings quality control chart. If the deviation $\%$ is between $\mathrm{X} 1$ and $\mathrm{X} 2$, it is in control. Otherwise it is out of control. Result -two groups of data show difference in the two different methods, fixed range method and LeveyJennings method. The two methods have the similar out-of-control ratio in negative control and positive control. But fixed range method is better than Levey-Jennings method in weak positive control. It has the statistical significance. Conclusion -- when the two methods are used for anti$\mathrm{HBc}$ detection, fixed range method and Levey-Jennings method show difference in weak positive true out-of-control ratio. Fixed range method is more obvious, sensitive and effective.
\end{abstract}

Keywords: Fixed range method, Enzyme linked immunosorbent assay, Internal quality control, Levey-Jennings quality control

\section{Introduction}

Enzyme linked immunosorbent assay (ELISA), a common immunodetection method, occupies a very important position in medical laboratory detection. No accepted ELISA internal quality control method has been widely recognized and applied. The reported quality control methods include instant method, L-J quality control method ${ }^{[1]}$, double reaction method ${ }^{[2]}$, critical value method, multiple-rule method etc. There are two methods used in clinical ELISA at home and abroad, quantitative L-J quality control method and qualitative double reaction method. There are many obvious problems when these methods are really used in qualitative ELISA experiment. ELISA experiment quality control methods used in domestic laboratory are not optimistic. More work needs to be done for the reasonable quality control plan to guarantee the accurate and reliable detection result and save quality control and reagent cost. Fixed range method ${ }^{[3]}$ contains the advantages of various methods and overcomes some disadvantages. The method is so easy that it can monitor the sensitivity, specificity, accuracy and effectiveness of the experiment. Besides, the experiment situation shall be recorded in a quality control chart. The author also compares the study method and the common method in quality control practice.

\section{Object and method}

Object: The specimen is from the internal quality control data of hepatitis B core antibody during the 16 months (from October 2015 to February 2017) in the immune group, the clinical lab, Jilin City Center Hospital.

Instrument and reagent instrument: DNX-9620A plate washer, DNX-9602G enzyme analyzer of Beijing Prang New Technology Co., Ltd, Beijing Prang New Technology C., Ltd. Reagent core antibody detection kit (euzymelinked immunosorbent assay) of hepatitis B virus, Beijing Wantai Biological Pharmacy Enterprise Co., Ltd, core antibody detection quality control product of hepatitis B, Zhengzhou Antu Biological Engineering Co. Ltd. 
Detection method fixed range method

Control test value (x): quality control product test value adopts $\mathrm{S} / \mathrm{CO}$ value records. Test value shall be converted into deviation $\%$ and drawn on the quality control chart.

Establishment of control limit: it adopts the fixed range method and sets upper control limit X2 and lower control limit X1 only. One of the lines adopts the positive judgement dividing value ${ }^{(4)}$. For example, if the two half-and-half sandwich method adopts $\mathrm{S} / \mathrm{CO} \geq 1$ as positive, 1 is the lower limit of weak positive quality control product. If the competition method adopts $\mathrm{S} / \mathrm{CO}<1$ as positive, 1 is the upper limit of weak positive quality control product. Another line can be set based on the quality objective and imprecision of the experiment. Generally, this line shall be set as $\mathrm{X}+3 \mathrm{SD}$ or $\mathrm{X}-3 \mathrm{SD}$ of $\mathrm{S} / \mathrm{CO}$ value when the quality control product is stable.

Control chart drawing: abscissa of fixed range quality control chart is batch or date. Its ordinate is deviation\%. Three lines are drawn on the coordinate properly: upper control limit X2, center line X and lower control limit X1. $\mathrm{X} 1$ is $0 \%$ and $\mathrm{X} 2$ is $100 \%$. Observed value description adopts $\mathrm{S} / \mathrm{CO}$ value or OD value. The relative deviation $\%$ of each observed value shall be calculated for the lower control limit. Deviation $\%=\left(\mathrm{X}_{\text {observed value }} \mathrm{X} 1\right) /(\mathrm{X} 2$ $\mathrm{X} 1) \times 100 \%$. Observed values of four control products shall be drawn on a quality control chart (see chart 1) based on deviation\%. Quality control chart adopts LIS quality control software or Excel software to draw the quality control chart.

Out-of-control judgement: if deviation\& is between X1 and X2, it is in control. Otherwise it is out of control.

Set the out-of-control rule of two methods

(1) Set the negative out-of-control rule of fixed range: 1 is control lower limit X1 of negative quality control product. $\mathrm{X}+3 \mathrm{SD}$ is control upper limit $\mathrm{X} 2$ of negative quality control product. Set the weak positive out-of-control rule of fixed range: X-3SD is lower limit X1 of weak positive quality control product; 1 is control upper limit of weak positive quality control product X2. Set the positive out-of-control rule of fixed range: 0 is lower limit X1 of positive quality control product; 1 is control upper limit X2 of positive quality control product. Out-of-control judgement: if it exceeds control lower limit X1 or control upper limit X2, it shall be out of control.

(2) Levey-Jennings quality control chart

Negative, positive, weak positive quality control rule: $1_{3 \mathrm{~s}}$, out-of-control judgement. If it exceeds $\pm 3 \mathrm{SD}$, it shall be out of control.

\section{Statistics analysis}

SPSS17.0 software is used for analysis. Data which follows the normal distribution shall have $\mathrm{t}$ experiment based on two groups, mean \pm standard deviation (expressed by $\mathrm{x} \pm \mathrm{s}$ ). Enumeration data in the experiment adopts $\mathrm{x}^{2}$ detection for data comparison. If $\mathrm{p}<0.01$, it has the statistical significance.

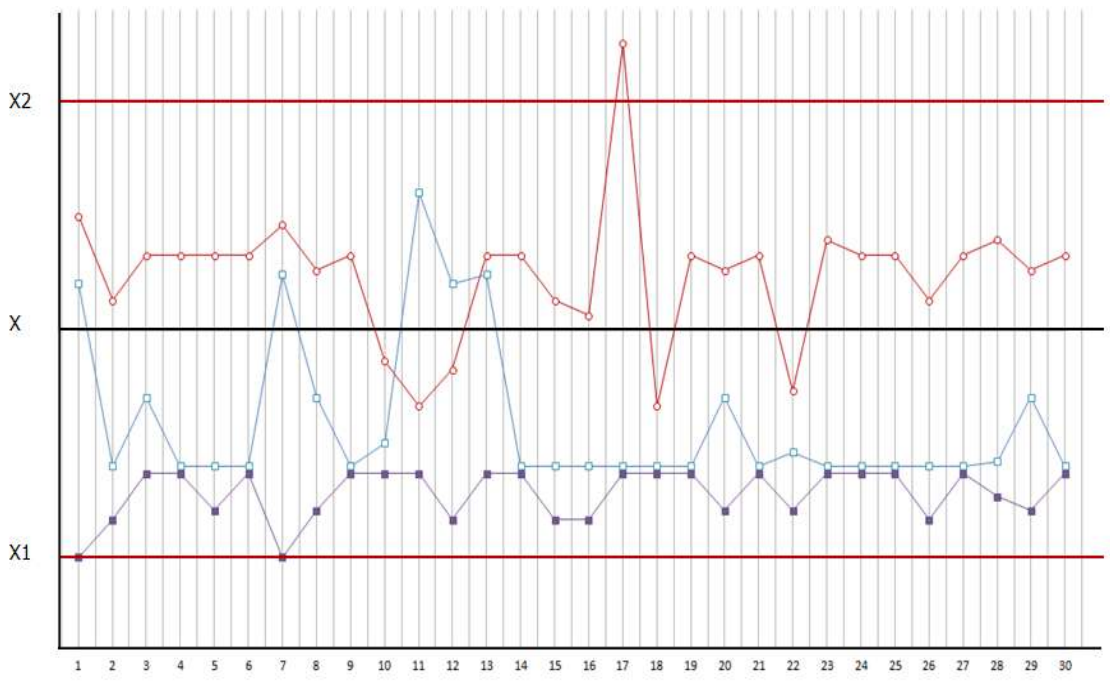

Fig. 1 fixed range method quality control

\section{Result}

True and false out-of-control ratio difference analysis of two methods

Result: the out-of-control ratio difference of the two methods has statistical significance. Namely, the two methods have different out-of-control ratios. Levey-Jennings method has $24.7 \%$ of true outof-control ratio. Fixed range method has $100.0 \%$ of true out-of-control ratio. See table 1 . 
Table 1 out-of-control difference analysis of two control methods

\begin{tabular}{lcccc}
\hline \multicolumn{1}{c}{$\begin{array}{c}\text { False } \\
\text { Experiment groups }\end{array}$} & $\begin{array}{c}\text { out-of- } \\
\text { control }\end{array}$ & $\begin{array}{c}\text { True } \\
\text { control }\end{array}$ & out-of- & Difference detection \\
\hline $\begin{array}{l}\text { Levey-Jennings } \\
\text { method }\end{array}$ & 61 & 20 & $\chi^{2}=38.031^{* *}$ \\
$\quad$ Fixed range method & 0 & 20 & $\boldsymbol{p}=0.000^{* *}$ \\
\hline
\end{tabular}

Note: ** means $\boldsymbol{p}<0.01$, and there exists obvious difference.

Negative true and false out-of-control ratio difference analysis of the two methods

Result: the true out-of-control ratio difference of the two methods has no statistical significance. Namely, the old method and the new method have the same negative true out-of-control ratio. See table 2 .

Table 2 negative out-of-control difference analysis of two control methods

\begin{tabular}{cccc}
\hline Experiment groups & False out-of-control & $\begin{array}{c}\text { True out-of- } \\
\text { control }\end{array}$ & Difference detection \\
\hline Levey-Jennings method & 3 & 5 & $\chi^{2}=0.783^{* *}$ \\
Fixed range method & 0 & 5 & $\boldsymbol{p}=\mathbf{0 . 3 7 6}$ \\
\hline
\end{tabular}

Note: ${ }^{* *}$ means $\boldsymbol{p}>0.01$, and there exists no difference.

Weak positive true and false out-of-control ratio difference analysis of the two methods

Result: the weak positive true out-of-control ratio difference of the two methods has statistical significance. Namely, the two methods have different weak positive true and false out-of-control ratios. Levey-Jenning method has $20.5 \%$ of true out-of-control ratio. Fixed range method has $100 \%$ of true out-of-control ratio. Levey-Jenning method has $79.5 \%$ of false out-of-control ratio. Fixed range method has $0.0 \%$ of true out-of-control ratio. See chart 3 .

Table 3 weak positive out-of-control difference analysis of the two control methods

\begin{tabular}{cccc}
\hline $\begin{array}{c}\text { Experiment } \\
\text { groups }\end{array}$ & $\begin{array}{c}\text { False out-of- } \\
\text { control }\end{array}$ & $\begin{array}{c}\text { True out- } \\
\text { of-control }\end{array}$ & Difference detection \\
\hline $\begin{array}{c}\text { Levey- } \\
\text { Jennings } \\
\text { method }\end{array}$ & 58 & 15 & $\chi^{2}=34.959^{* *}$ \\
$\begin{array}{c}\text { Fixed range } \\
\text { method }\end{array}$ & 0 & 15 & $\boldsymbol{p}=0.000$ \\
\hline Note: ** means $\boldsymbol{p}<0.01$, and there exists obvious difference.
\end{tabular}

Positive true and false out-of-control ratio difference analysis of the two methods

Result: there is no positive true and false out-of-control case of the two methods. The two methods have the similar positive out-of-control ratio effect. See chart 4.

Table 4 negative out-of-control difference analysis of the two control methods

\begin{tabular}{ccc}
\hline Experimental groups & False out-of-control & $\begin{array}{c}\text { True out-of- } \\
\text { control }\end{array}$ \\
\hline Levey-Jennings method & 0 & 0 \\
Fixed range method & 0 & 0 \\
\hline
\end{tabular}

\section{Discussion}


Control lower limit of negative quality control new quality control method is set as positive judgement dividing value 1. It effectively guarantees the negative and positive judgement effectiveness in ELISA experiment ${ }^{[4]}$. Lower limit of L-J quality control is X-3SD. So control lower limit value of L-J quality control can be higher than positive judgement value 1 or lower than positive judgement value 1 . If L-J quality control lower limit is higher than 1 , the quality control value between X-3SD and 1 -- L-J quality control shall be judged as out-of-control. Thus it leads to false out-of-control. Because negative quality control product is judged as negative. The experiment is effective. If L-J quality control lower limit is lower than 1, the quality control value between X3SD and 1 -- L-J quality control shall be judged as in-control. But the negative result is made to be positive. And the experiment is invalid. But the out-of-control result fails to be detected by L-J quality control. The study result shows $79.5 \%$ of false out-of-control in the weak positive quality control close to positive judgement value 1, which affects the effectiveness of the experiment. At the same time, there exists no difference in the negative true and false out-of-control ratio difference analysis of the two methods. The two methods adopt X+3SD control limit in terms of negative quality control upper limit. Therefore, they are equivalent in upper limit error detection ratio and false out-of-control ratio ${ }^{\text {[5] }}$. Control upper limit of weak positive quality control new quality control method shall be set as positive judgement dividing limit 1. Experiment effectiveness judgement standard of new quality control must be made to be positive rather than negative. Upper limit of L-J quality control is $\mathrm{X}+3 \mathrm{SD}$. When quality control value is between $\mathrm{X}+3 \mathrm{SD}$ and 1 , L-J quality control shall be judged as out-of-control. But the experiment is effective in terms of weak positive quality control negative positive judgement. There exists obvious difference in the weak positive true and false out-of-control ratio of the two methods. It shows L-J quality control has false out-of-control and excessive control. The study also analyzes the positive true and false out-ofcontrol ratio difference of the two methods. There exists no positive true and false out-of-control case of the two methods. The two methods are close in terms of control positive out-of-control ration result. The result shows that the two methods are equivalent in experiment error detection ratio and false out-of-control ratio. When L-J quality control lower limit is set as X-3SD, X-3SD $<0$ may be negative. The result can not be accepted. Because the detection data result is higher than 0 , it can not be negative. So it may reduce the effectiveness of Levey-Jennings method.

The choice of quality control rule. When the two quality control methods are compared in terms of the quality control effectiveness, L-J quality control only chooses the single $1_{3 S}$ out-of-control rule and its weak positive quality control shall be $79.5 \%$ of out-of-control. If $22_{2 \mathrm{~S}} / 10_{\mathrm{X}} / 4_{1 \mathrm{~S}} / 1_{3 \mathrm{~S}}$ and other quality control rules are included into L-J quality control, it shall increase the false out-ofcontrol probability and lead to excessive control. Thus staff need to deal with more false out-ofcontrol work. It also increases the quality control cost. In terms of quality control chart practicability, the quality control chart (see chart 1) of fixed range method is better than L-J quality control chart. The quality control chart of fixed range method is similar to the qualitative quality control chart ${ }^{\text {[6] }}$ of dual zone method. But it is superior to the qualitative quality control chart of dual zone method. L-J quality control chart has a problem that one concentration level of quality control data can only be drawn on an L-J quality control chart. Three concentration levels of quality control data need to be drawn on three L-J quality control charts. Different concentration levels of quality control data can be drawn on a quality control chart of the fixed range method. Certainly L-J quality control chart can adopt Z-grade method to draw different concentration levels of quality control data on a chart. When Z-grade is used, quality control rule must be chosen carefully to avoid false out-of-control and excessive control etc. The author shall not explain too much here. Fixed range method quality chart and L-J quality control chart are equivalent according to the quality control data fluctuation quality control data trend change on the quality control chart.

Levey-Jennings method needs to accumulate 20 pieces of quality control data for mean value and standard deviation calculation. In the past, instant method was used to control the first 20 data points. But instant method is greatly affected by previous results. It easily leads to sampling error ${ }^{\mathbf{1 7}, 8 \mathbf{1}}$. If previous results have higher degree of precision, it shall have higher requirement on the later data and false out-of-control ${ }^{\mathbf{1 9 1}}$ may occur. It shall have an influence on the later result 
judgement ${ }^{\mathbf{1 0 , 1 1}}$ or lead to inconsistency between in-control and quality control rule judgement result whether outlier is accepted or rejected. Namely, instant method can not show the trend. So instant method has its own defects. Besides, ELISA experiment has the function of negative and positive judgement. Fixed range method can use variable coefficient (or variable coefficient of kit) of previous batches as the temporary variable coefficient. Mean value shall be calculated in a short time as the temporary mean value. Quality control chart can be established. Target value and standard deviation of 20 pieces of data shall be changed. But ELISA reagent and quality control serum have a short period of validity. There exists batch difference. So a further study needs to be conducted to explore how ELISA experiment sets a more reasonable mean value and standard deviation.

\section{References}

[1] Wang Zhiguo, Clinical Detection Quality Control Technology, second version: People's Medical Publishing House, 2012:85-92

[2] Wang Zhiguo, Clinical Detection Quality Control Technology, Beijing: People's Medical Publishing House, 2008:267-270

[3] Wang Jiandong, Zhao Xiaomei, Xia Wei, Chinese Modern Drug Application [J].2009,3(9):189190.

[4] Li Jinming, Clinical Immunoassay Technology, Beijing: People's Military Medical Publishing House, 2005:93-105

[5] Wang Zhiguo, Clinical Detection Quality Control Technology, second version, Beijing: People's Medical Publishing House, 2012:167-169

[6] Zhou Youliang, SLISA Qualitative Quality Control Chart Design, Travel Medicine Science [J].2008,6(14):39-41

[7] Gui Ping, Zhang Ting, Wang Deqing, Comparison of Instant Method and Levey-Jennings Method in ELISA Detection HBsAg [J], Mark Immune Analysis and Clinic, 2016,4(23):463-464

[8] Chen Fei, Instant Method Exploration and Improvement in Handwork ELISA Internal Quality Control Method [J], International Detection Medical Magazine 2011,11(32): 2242-2243

[9] Kong Wei, A Study on "Instant Method" Used in ELISA Experiment Detection HBsAg Internal Quality Control[J], Chinese Pharmaceutical Economics.2015,7:71-72

[10] Wu Qikang, Chen Binhong, Chen Zhanze etc, A Study on Grubbs Method Used in Hepatitis B Two Half-and-Half Internal Quality Control of Enzyme Linked Immunosorbent Assay [J], Detection Medicine and Clinic 2015,7 (12):2088-2089

[11] Mei Chuanliang, Du Yong, A Study on the First Three Points of ELISA "Instant Method" Quality Control Chart [J], Chinese Journal of Blood Transfusion .2012,(25):71

[12] Guo Tao, ELISA Blood Detection Result Judgement Based on Comparison Between Instant Method Quality Control and Levey-Jennings Chart Quality Control Method [J], Modern Preventive Medicine 2010,(37):897-899 